\title{
Stability Analyses of Static Greedy Link Schedulings in MC-MR Wireless Networks
}

\author{
Peng-Jun Wan*, Zhiguo Wan ${ }^{\dagger}$, Zhu Wang*, Xiao-Hua Xu*, Shaojie Tang*, and Xiaohua Jia ${ }^{\ddagger}$ \\ * Department of Computer Science, Illinois Institute of Technology \\ Emails: wan@cs.iit.edu,zwang59@iit.edu,xxu23@iit.edu, stang7@iit.edu \\ $\dagger$ School of software, Tsinghua University \\ Email:wanzhiguo@tsinghua.edu.cn \\ $\ddagger$ Department of Computer Science, City University of Hong Kong \\ Email: csjia@cityu.edu.hk
}

\begin{abstract}
Static greedy link schedulings have much simpler implementation than dynamic greedy link schedulings such as Longest-queue-first (LQF) link scheduling. However, its stability performance in multi-channel multi-radio (MC-MR) wireless networks is largely under-explored. In this paper, we present a stability subregion with closed form of a static greedy link scheduling in MC-MR wireless networks under the 802.11 interference model. By adopting some special static link orderings, the stability subregion is within a constant factor of the stable capacity region of the network. We also obtain constant lower bounds on the throughput efficiency ratios of the static greedy link schedulings in some special static link orderings.
\end{abstract}

Keywords-Stability, multi-channel multi-radio, link scheduling.

\section{INTRODUCTION}

With the rapid technology advances, many off-the-shelf wireless transceivers (i.e., radios) are capable of operating on multiple channels. For example, the IEEE $802.11 \mathrm{~b} / \mathrm{g}$ standard and IEEE 802.11a standard provide 3 and 12 channels respectively, and MICA2 sensor motes support more than 50 channels. The rapidly diminishing prices of the radios has also made it feasible to equip a wireless node with multiple radios. Providing each node with one or more multi-channel radios offers a promising avenue for enhancing the network capacity by simultaneously exploiting multiple non-overlapping channels through different radio interfaces and mitigating interferences through proper channel assignment. In this paper, we take a queuing-theoretic study of wireless link scheduling in multichannel multi-radio (MC-MR) wireless networks under the 802.11 interference model. A wireless link scheduling algorithm takes as an input a set of communication links together with their backlogs of packets waiting for transmission, and outputs a set of packets which can be transmitted successfully at the same time.

We assume that time is slotted. For each $t \in \mathbb{N}$, the $t$-th time slot is the time interval $(t-1, t]$. Any packet arriving in a slot is assumed to arrive at the end of the slot, and may only be transmitted in the subsequent slots. In addition, the packet arrivals are assumed to be mutually independent and temporally i.i.d. processes with arrival rate vector $\alpha$. Consider a wireless link scheduling algorithm $\mathcal{A}$. In each time-slot, a set of backlogged packets are selected to be transmitted using the algorithm $\mathcal{A}$. Let $X(t)$ (respectively, $Y(t)$ ) denote the vector of cumulative number of packets arriving (respectively, transmitted) in the first $t$ time slots, and $Z(t)$ denote the vector of number of packets queued at the very end of time slot $t$. Then,

$$
Z(t)=Z(0)+X(t)-Y(t) .
$$

The network is said to be stable if the Markov chain $(Z(t))$ is positive recurrent. The stability region of the wireless link scheduling algorithm $\mathcal{A}$, denoted by $\Lambda$, is the set of arrival rate vectors $\alpha$ such that the network is stable. Let $P$ be the maximum stability region of the network, which consists of arrival rate vectors such that there exists a scheduling policy stabilizing the network. The efficiency ratio of the wireless link scheduling algorithm $\mathcal{A}$ is defined to be

$$
\sup \left\{\sigma \in \mathbb{R}_{+}: \sigma P \subseteq \Lambda\right\} .
$$

The seminal work of Tassiulas and Ephremides [13] provided a scheduler with efficiency ratio equal to one. However, this optimal scheduler has exponential running time in general. Thus, a class of greedy link scheduling (GLS) algorithms that trade-off throughput efficiency for implementation simplicity have been proposed in single-channel single-radio (SC-SR) wireless networks [2], [4], [5], [6], [10], [19], [20] as well as in MC-MR wireless networks [9], [1], [18]. Consider a link ordering $\prec$. The algorithm GLS in $\prec$ schedules the transmissions by all communication links along the ordering $\prec$ in the following greedy manner: Each link transmits as many packets as possible from its queue using the radios at its two endpoints which have not been used by any preceding links and the channels which have not been used by any preceding conflicting links. Among the class of GLS algorithms, 
there is a further trade-off between throughput efficiency and simplicity A dynamic GLS adopts a link ordering $\prec$ which may vary with the queue lengths of the links; and a static GLS adopts a link ordering $\prec$ which does not vary with the queue lengths of the links. A renowned dynamic GLS is the Longest-Queue-First (LQF) scheduling, which adopts the link ordering in the decreasing order of queue lengths. A dynamic GLS requires the expensive recomputation of the link ordering in each time-slot, and in return it may achieve better throughput efficiency; on the other hand, a static GLS only needs a single pre-computation of the link ordering and therefore enjoys much simpler implementation. The stability of the LQF scheduling in both SC-SR wireless networks [4], [5], [6], [16] and MC-MR wireless networks [18] is now well understood. However, the stability of static GLS algorithms in both SC-SR wireless networks and MCMR wireless networks has been under-studied. As the result, the quantitative characterization of the trade-off between the throughput efficiency and implementation simplicity of GLS algorithms is still missing.

Contributions of this paper: The first main contribution of this paper is a stability subregion of a static GLS with closed form. Such stability subregion is shown to be within a constant factor of the maximum stability region of the network if some special static link orderings are adopted. In addition, it can be checked in polynomial time whether a given vector of packet arrival rates lies in this stability subregion. This computational tractability is particularly favorable for crosslayer optimization, where one needs to allocate the link rates efficiently while still ensuring the network stability under a static GLS. The second main contribution of this paper is the discovery of constant lower bounds on the throughput efficiency ratios of the static GLS in some special link orderings. By comparing the stable capacity subregion and the throughput efficiency ratios of the static GLS against those of the LQF scheduling, we derive the quantitative trade-off between the throughput efficiency and implementation simplicity of GLS algorithms. Based on such quantitative trade-off, the static GLS is advocated in most networking settings.

Related works: The throughput efficiency ratio of the LQF scheduling in SC-SR was fully characterized by Joo et al. [5], [6]. Built upon the prior works by Dimakis and Walrand [4] which presented sufficient conditions for $\mathbf{L Q F}$ to achieve $100 \%$ throughput, Joo et al. [5], [6] proved that the throughput efficiency ratio of $\mathbf{L Q F}$ is exactly the local pooling factor (LPF) of the conflict graph of the communication links. The LPF is a pure graph-theoretic parameter. Thus, the works by Joo et al. [5], [6] built an elegant bridge between a queuingtheoretic parameter and a graph-theoretic parameter. Under the 802.11 interference model with uniform interference radii, the LPF is shown to be at least $1 / 6$ in [6]. Sparked by the works in [6], Leconte el al. [7] and Li el al. [8] presented some properties of LPF. Leconte el al. [7] derived tighter lower bounds on LPF in networks of size at most 28 under the 802.11 interference model with uniform interference radii. $\mathrm{Li}$ el al. [8] gave an alternative definition of LPF and also introduced a refined notion of LPF. Wan et al. [16] further proved that the LPF is at least $1 / 16$ under the 802.11 interference model with arbitrary interference radii. However, it remains computationally intractable to decide whether a given vector of packet arrival rates meets the so-called local-pooling condition.

The stability of the LQF scheduling in MC-MR wireless networks has been well-studied in [9], [1], [18]. Lin and Rasool [9] derived a lower bound $1 / 10$ on the throughput efficiency ratio of the $\mathbf{L Q F}$ scheduling under the 802.11 interference model with uniform interference/communication radii. Brzezinski et al. [1] considered the variant of the $\mathbf{L Q F}$ scheduling with (temporarily) static channel assignment and the only interference assumed was the primary interference, but no analytical bounds on the throughput efficiency ratio were provided. Recently, a stability subregion of LQF with closed form was given in [18]. Such stability subregion is shown to be within a constant factor of the maximum stability region of the network. In addition, constant lower bounds on the throughput efficiency ratios of the $\mathbf{L Q F}$ scheduling were derived in [18]. Specifically, the throughput efficiency ratio of the $\mathbf{L Q F}$ scheduling is at least $1 / 8$ under the 802.11 interference model with uniform interference radii, at least $1 / 20$ under the 802.11 interference model with arbitrary interference radii.

In SC-SR wireless networks under the 802.11 interference model with uniform interference radii, the throughput efficiency ratio of a static GLS in an arbitrary link ordering was shown to be at least $1 / 8$ in [2], [19], [20]. The technical approach in [2], [19], [20] is quite different from the approach followed in this paper. In fact, the lower bound $1 / 8$ can be derived in a simpler manner by using the fact that any static GLS is actually a 8-approximation algorithm for MaximumWeight Independent Set under the 802.11 interference model with uniform interference radii. Furthermore, the work in this paper implies a stronger lower bound $1 / 6$ on the the throughput efficiency ratio of a static GLS under the same networking setting.

The remainder of this paper is organized as follows. Section II introduces some basic results from functional analysis and probability theory. Section III defines the stability region of a MC-MR wireless network. Section IV presents a stability subregion of a static GLS. Section V derives the lower bounds on the throughput efficiency ratios of the static GLS in some special static link orderings. scheduling. Finally, we conclude this paper in Section VI by comparing the stability performances of the static GLS in some special static link orderings and the $\mathbf{L Q F}$ scheduling. 


\section{PRELIMINARIES}

Let $I$ be an interval in the real line $\mathbb{R}$. A function $f: I \rightarrow \mathbb{R}$ is absolutely continuous on $I$ if for every $\varepsilon>0$, there is a $\delta>0$ such that whenever a finite sequence of pairwise disjoint sub-intervals $\left[s_{k}, t_{k}\right]$ of $I$ satisfies

$$
\sum_{k}\left|s_{k}-t_{k}\right|<\delta
$$

then

$$
\sum_{k}\left|f\left(s_{k}\right)-f\left(t_{k}\right)\right|<\varepsilon
$$

If $f$ is absolutely continuous, then $f$ has a derivative $f^{\prime}$ almost everywhere; the points at which $f$ is differential are called the regular points of $f$. The following property of absolutely continuous functions is implicitly used in [3].

Lemma 2.1: Let $f$ be an absolutely continuous nonnegative function on $\mathbb{R}_{+}$and $\kappa$ be a positive constant. Suppose that for every almost every regular point $t, f^{\prime}(t) \leq-\kappa$ whenever $f(t)>0$. Then, $f$ is non-increasing, and once it reaches zero it stays zero forever. Moreover, $f(t)=0$ for all $t \geq f(0) / \kappa$.

A function $f$ on $\mathbb{R}_{+}$is said to be Lipschitz continuous with Lipschitz constant $C$ (or simply $C$-Lipschitz continuous) for some constant $C>0$ if for any $s, t \in \mathbb{R}_{+}$,

$$
|f(s)-f(t)| \leq C|s-t|
$$

Lipschitz continuous functions are absolutely continuous. A function $f$ which takes values in $k$-dimensional Euclidean space is said to be absolutely (respectively, Lipschitz) continuous if each of its component is absolutely (respectively, Lipschitz) continuous. For any vector $x$ in an Euclidean Space, $\|x\|_{\infty}$ and $\|x\|_{1}$ denote the maximum norm (also called uniform norm) and the Manhattan norm of $x$ respectively.

Let $\left(f_{n}\right)$ be a sequence of functions on $\mathbb{R}_{+}$and let $f$ be a continuous function on $\mathbb{R}_{+}$. We say that $f_{n} \rightarrow f$ uniformly on compact sets, or simply $f_{n} \rightarrow f$ u.o.c., if for each $t>0$,

$$
\sup _{0 \leq s \leq t}\left|f_{n}(s)-f(s)\right| \rightarrow 0 .
$$

The following lemma was stated in Lemma 4.1 of [3].

Lemma 2.2: Let $\left(f_{n}\right)$ be a sequence of non-decreasing real-valued functions on $\mathbb{R}_{+}$, and $f$ be a continuous function on $\mathbb{R}_{+}$. Assume that $\left(f_{n}\right)$ converges pointwise to $f$. Then the convergence is u.o.c.

The following theorem on the convergence of random variables is stated in Theorem 2.2.3 of [12].
Theorem 2.3: Suppose that a sequence of random variables $\left(\xi_{n}\right)$ converge to a random variable $\xi$ in probability.

1) If $\xi_{n}$ is uniformly integrable, then $\mathbf{E}[|\xi|]<\infty$ and $\lim _{n \rightarrow \infty} \mathbf{E}\left[\xi_{n}\right]=\mathbf{E}[\xi]$.

2) If $\xi_{n} \geq 0, \mathbf{E}[\xi]<\infty$, and $\lim _{n \rightarrow \infty} \mathbf{E}\left[\xi_{n}\right]=\mathbf{E}[\xi]$, then $\xi_{n}$ is uniformly integrable.

\section{Network Stability Region}

Consider an instance of MC-MR multihop wireless network with a set $V$ of networking nodes and a set $A$ of nodelevel communication links. Each node $v$ has $\tau(v)$ radios, and there are $\lambda$ non-overlapping channels. In the fine-grained network representation [15] of the MC-MR wireless network, each communication link is encoded by an ordered quintuple specifying the transmitting node, the receiver node, the radio at the transmitting node, the radio at the receiving node, and the channel. Specifically, for each node-level link $(u, v)$ in $A$, we make $\lambda \cdot \tau_{u} \cdot \tau_{v}$ replications $(u, v, i, j, k)$ for $1 \leq i \leq \tau_{u}$, $1 \leq j \leq \tau_{v}$, and $1 \leq k \leq \lambda$. A replication $(u, v, i, j, k)$ always utilizes the $i$-th radio at $u$ and the $j$-th radio at $v$ over the $k$-th channel. For each subset $B$ of $A$, we use $B^{\tau, \lambda}$ to denote the set of all replications of the links in $B$. In particular, $A^{\tau, \lambda}$ is the set of all replicated links of the links in $A$. A subset $I$ of $A^{\tau, \lambda}$ can transmit at the same time if and only if (1) all replication links in $I$ are radio-disjoint, in other words, no pair share a common radio, and (2) for each channel $k$, all the replication links in $I$ transmitting over channel $k$ are conflict-free. Let $\mathcal{I}^{\tau, \lambda}$ denote the collection of the subsets of $A^{\tau, \lambda}$ which can transmit successfully at the same time. For each $I \in \mathcal{I}^{\tau, \lambda}$, its service rate is the vector $d \in \mathbb{R}_{+}^{A}$ given by

$$
d_{a}=\left|I_{j} \cap\{a\}^{\tau, \lambda}\right|
$$

for each $a \in A$.

A set

$$
\Pi=\left\{\left(I_{j}, \ell_{j}\right) \in \mathcal{I}^{\tau, \lambda} \times \mathbb{R}_{+}: 1 \leq j \leq m\right\}
$$

is called a (fractional) link schedule of some $d \in \mathbb{R}_{+}^{A}$ if

$$
d_{a}=\sum_{j=1}^{m} \ell_{j}\left|I_{j} \cap\{a\}^{\tau, \lambda}\right|
$$

for each $a \in A$. The two values $m$ and $\sum_{j=1}^{m} \ell_{j}$ are referred to as the size and length (or latency) of $\Pi$ respectively. For any $d \in \mathbb{R}_{+}^{A}$, the minimum latency $\chi^{*}(d)$ of $d$ is defined as the minimum length of all fractional link schedules of $d$. The stability region of the MC-MR wireless network is

$$
P=\left\{d \in \mathbb{R}_{+}^{A}: \chi^{*}(d)<1\right\} .
$$

Consider a link $a \in A$. An independent set $I \in \mathcal{I}^{\tau, \lambda}$ is said to be a-tight if each link $e \in\{a\}^{\tau, \lambda} \backslash I$ has a conflict with 
some link in $I \cap N_{G}^{\prec}(a)^{\tau, \lambda}$. We use $\Phi_{a}$ to denote the convex hull of service rates of the $a$-tight independent sets $\mathcal{I}^{\tau, \lambda}$. By Lemma 2.4 in [21], for any $a \in A$ and $d \in \Phi_{a}$,

$$
\Gamma\left(N_{G}^{\prec}(a), a ; d\right) \geq 1 .
$$

\section{Stability Subregion}

Consider an instance of MC-MR wireless network specified in Section III. Two links in $A$ are said to have a conflict if they cannot transmit at the same time over the same channel. Furthermore, a conflicting pair of distinct links in $A$ are said to have primary conflict if there share one common end, and secondary conflict otherwise. For the sake of convenience, each link is said to have a self-conflict with itself. The concise conflict graph [21] of the MC-MR wireless network is the edge-weighted graph $G$ on $A$ in which there is an edge between each conflicting pair of links $(a, b)$ whose weight denoted by $c(a, b)$, is defined as follows:

- If $b=a$ (i.e., self-conflict), then

$$
c_{a, b}=1-\left(1-\frac{1}{\tau_{u}}\right)\left(1-\frac{1}{\tau_{v}}\right)\left(1-\frac{1}{\lambda}\right)
$$

where $u$ and $v$ are the two endpoints of $a$.

- If $a$ and $b$ have a common endpoint $u$ (i.e., $a$ and $b$ have a primary conflict), then

$$
c_{a, b}=1-\left(1-\frac{1}{\tau_{u}}\right)\left(1-\frac{1}{\lambda}\right) .
$$

- If $a$ and $b$ have the secondary conflict, then

$$
c_{a, b}=\frac{1}{\lambda} .
$$

Note that $c(a, b)=c(b, a)$. Let $\mathcal{I}$ denote the collection of the independent sets in $G$. In other words, $\mathcal{I}$ is the collection of the subsets of $A$ which can transmit successfully at the same time over the same channel. Note that $G$ can be regarded as a generalization of the conventional conflict graph of the underlying SC-SR wireless network by adding a self-loop at each link and assigning each edge a weight specified by the function $c$. Thus, $\mathcal{I}$ is essentially the collection of the independent sets of links in the underlying SC-SR wireless network.

For any link $a \in A, N_{G}(a)$ denotes the set of neighbors of $a$ in $G$. Since $G$ has a self-loop at each vertex, $a$ is a neighbor to itself, and hence $a \in N_{G}(a)$. Thus, $N_{G}(a)$ consists of all links in $A$ (including itself) having conflict with $a$. For any link $a$, any subset $B$ of links, and any $d \in \mathbb{R}_{+}^{A}$, define

$$
\Gamma(B, a ; d)=\sum_{b \in N_{G}(a) \cap B} c_{a, b} d_{b} .
$$

Consider a link ordering $\prec$ of $A$. For any link $a \in A, N_{G}^{\prec}(a)$ denotes the set of neighbors of $a$ in $G$ preceding $a$ in the ordering $\prec$ plus $a$ itself. For any $d \in \mathbb{R}_{+}^{A}$, the value

$$
\max _{a \in A} \Gamma\left(N_{G}^{\prec}(a), a ; d\right)
$$

is referred to as $d$-weighted inductivity of $\prec$ and is denoted by $\Delta^{\prec}(d)$. Let

$$
Q^{\prec}=\left\{d \in \mathbb{R}_{+}^{A}: \Delta^{\prec}(d)<1\right\} .
$$

Theorem 4.1: $Q^{\prec}$ is a stability subregion of the greedy link scheduling in $\prec$.

We shall prove Theorem 4.1 by applying the MalyshevMenshikov Criterion [11] for ergodicity of discrete-time countable-state Markov chains. For any $n \in \mathbb{N}$, we denote by $Z^{(n)}(t)$ (respectively, $X^{(n)}(t), Y^{(n)}(t)$ ) the vector of queue length (cumulative number of arriving packets, cumulative number of transmitted packets) in a system at the end of timeslot $t$ with its initial total queue length $\left\|Z^{(n)}(0)\right\|_{1}=n$. Let

$$
T=\left\lceil\sum_{j=1}^{|A|} \frac{1}{\left(1-\Delta^{\prec}(\alpha)\right)^{j}}\right\rceil .
$$

By the Malyshev-Menshikov Criterion [11], Theorem 4.1 follows immediately from the theorem below.

Theorem 4.2: For any $\alpha \in Q^{\prec}$,

$$
\lim _{n \rightarrow \infty} \mathbf{E}\left[\left\|\frac{Z^{(n)}(n T)}{n}\right\|_{1}\right]=0 \text {. }
$$

The proof of Theorem 4.2 utilizes Theorem 2.3. By the strong law of large numbers,

$$
\left\|\frac{X^{(n)}(n T)}{n}\right\|_{1}=T\left\|\frac{X^{(n)}(n T)}{n T}\right\|_{1} \rightarrow T\|\alpha\|_{1}
$$

almost surely, and

$$
\begin{aligned}
\mathbf{E}\left[\left\|\frac{X^{(n)}(n T)}{n}\right\|_{1}\right] & =T \cdot \mathbf{E}\left[\left\|\frac{X^{(n)}(n T)}{n T}\right\|_{1}\right] \\
& =T\|\alpha\|_{1} .
\end{aligned}
$$

By Theorem 2.3, the sequence $\left(\left\|\frac{X^{(n)}(n T)}{n}\right\|_{1}\right)$ is uniformly integrable. Since

$$
\left\|\frac{Z^{(n)}(n T)}{n}\right\|_{1} \leq\left\|\frac{X^{(n)}(n T)}{n}\right\|_{1},
$$

the sequence $\left(\left\|\frac{Z^{(n)}(n T)}{n}\right\|_{1}\right)$ is also uniformly integrable. Again, by Theorem 2.3, Theorem 4.2 would hold if

$$
\left\|\frac{Z^{(n)}(n T)}{n}\right\|_{1} \rightarrow 0
$$


in probability. We will actually prove a stronger result that

$$
\left\|\frac{Z^{(n)}(n T)}{n}\right\|_{1} \rightarrow 0
$$

almost surely. Consider a sample path (i.e. realization) $\omega$ of

$$
\left(Z^{(n)}(0): n \in \mathbb{N}\right) \cup\left(X^{(n)}(t): n, t \in \mathbb{N}\right) \text {. }
$$

It is is said to be well-behaved if

$$
\lim _{t \rightarrow \infty} \frac{X^{(n)}(t, \omega)}{t}=\alpha .
$$

By the strong law of large numbers, every sample path is almost surely well-behaved. We will prove that for any wellbehaved sample path $\omega$,

$$
\left\|\frac{Z^{(n)}(n T, \omega)}{n}\right\|_{1} \rightarrow 0
$$

from which we can conclude that

$$
\left\|\frac{Z^{(n)}(n T)}{n}\right\|_{1} \rightarrow 0
$$

almost surely, and hence Theorem 4.2 holds.

Fix a well-behaved sample path $\omega$. Denote $X^{(n)}(t, \omega)$ (respectively, $Y^{(n)}(t, \omega), Z^{(n)}(t, \omega)$ ) by $x^{(n)}(t)$ (respectively, $\left.y^{(n)}(t), z^{(n)}(t)\right)$. Then, all of them are deterministic. In order to show that

$$
\left\|\frac{z^{(n)}(n T)}{n}\right\|_{1} \rightarrow 0
$$

it is sufficient to show that for any infinite increasing sequence $S$ of positive integers, there is an infinite subsequence $S^{\prime}$ of $S$ along which

$$
\left\|\frac{z^{(n)}(n T)}{n}\right\|_{1} \rightarrow 0
$$

So, we further fix an infinite increasing sequence $S$ of positive integers. For convenience, we define $x^{(n)}(0)$ and $y^{(n)}(0)$ to be the vector of zeros. We extend $x^{(n)}(t)$ (respectively, $\left.y^{(n)}(t), z^{(n)}(t)\right)$ to all non-negative real numbers by linear interpolation. Then, for any $t \geq 0$ and any $n \in \mathbb{N}$,

$$
\frac{z^{(n)}(n t)}{n}=\frac{z^{(n)}(0)}{n}+\frac{x^{(n)}(n t)}{n}-\frac{y^{(n)}(n t)}{n} .
$$

The following property of the sequence $\left(x^{(n)}(t)\right)$ was proved in [18].

Lemma 4.3: For any $t \geq 0, \lim _{n} \frac{x^{(n)}(n t)}{n}=\alpha t$.

For each $t \in \mathbb{N}$, let $I^{(n)}(t) \in \mathcal{I}^{\tau, \lambda}$ be the set of replicated links which are scheduled to transmit in the $t$-th time slot. Let $d^{(n)}(t) \in \mathbb{R}_{+}^{A}$ be the aggregation of $\mathbf{1}^{I^{(n)}(t)}$. The average service rate in a time interval $\left[t_{1}, t_{2}\right]$ is defined to be

$$
\bar{y}^{(n)}\left(t_{1}, t_{2}\right)=\frac{y^{(n)}\left(t_{2}\right)-y^{(n)}\left(t_{1}\right)}{t_{2}-t_{1}} .
$$

It has the following properties, which were proved in [18].

Lemma 4.4: Consider any $0 \leq t_{1}<t_{2}$.

1) For any $s=(1-\varepsilon) t_{1}+\varepsilon t_{2}$ for some $\varepsilon \in[0,1]$,

$$
\bar{y}^{(n)}\left(t_{1}, t_{2}\right)=\varepsilon \bar{y}^{(n)}\left(t_{1}, s\right)+(1-\varepsilon) \bar{y}^{(n)}\left(s, t_{2}\right) .
$$

2) $\bar{y}^{(n)}\left(t_{1}, t_{2}\right)$ is a convex combination of

$$
\left\{d^{(n)}(t):\left\lfloor t_{1}\right\rfloor+1 \leq t \leq\left\lceil t_{2}\right\rceil, t \in \mathbb{N}\right\} .
$$

3) $\left\|\bar{y}^{(n)}\left(t_{1}, t_{2}\right)\right\|_{\infty} \leq\|\tau\|_{\infty}$.

Note that for any $0 \leq t_{1}<t_{2}$,

$$
\begin{aligned}
& \frac{\frac{y^{(n)}\left(n t_{2}\right)}{n}-\frac{y^{(n)}\left(n t_{1}\right)}{n}}{t_{2}-t_{1}} \\
& =\frac{y^{(n)}\left(n t_{2}\right)-y^{(n)}\left(n t_{1}\right)}{n t_{2}-n t_{1}} \\
& =\bar{y}^{(n)}\left(n t_{1}, n t_{2}\right) .
\end{aligned}
$$

By the third part of Lemma 4.4, $\frac{y^{(n)}(n t)}{n}$ is $\|\tau\|_{\infty}$-Lipschitz continuous, and hence is equicontinuous. By the Arzela-Ascoli theorem, there is an infinite subsequence $S_{1}$ of $S$ along which $\frac{y^{(n)}(n t)}{n}$ converges to some function $\beta(t)$. In addition, $\beta(t)$ is also $\|\tau\|_{\infty}$-Lipschitz continuous. Since

$$
\left\|\frac{z^{(n)}(0)}{n}\right\|_{1}=1
$$

for any $n \in \mathbb{N}$, there is an infinite subsequence $S^{\prime}$ of $S_{1}$ along which $\frac{z^{(n)}(0)}{n}$ converges. Therefore, along the sequence $S^{\prime}$, $\frac{z^{(n)}(0)}{n}, \frac{x^{(n)}(n t)}{n}$ and $\frac{y^{(n)}(n t)}{n}$ all converge. Since both $\frac{x^{(n)}(n t)}{n}$ and $\frac{y^{(n)}(n t)}{n}$ are increasing function of $t$ for each $n$, they converge u.o.c along $S^{\prime}$ to $\alpha t$ and $\beta(t)$ respectively by Lemma 2.2. As

$$
\frac{z^{(n)}(n t)}{n}=\frac{z^{(n)}(0)}{n}+\frac{x^{(n)}(n t)}{n}-\frac{y^{(n)}(n t)}{n},
$$

$\frac{z^{(n)}(n t)}{n}$ also converges u.o.c. along $S^{\prime}$ to some function $\gamma(t)$. Since

$$
\left\|\frac{z^{(n)}(0)}{n}\right\|_{1}=1
$$

$\|\gamma(0)\|_{1}=1$. In addition,

$$
\gamma(t)=\gamma(0)+\alpha t-\beta(t)
$$

Clearly, $\gamma(t)$ is also Lipschitz continuous with Lipschitz constant $\|\alpha\|_{\infty}+\|\tau\|_{\infty}$.

A time $t \in \mathbb{R}_{+}$is said to be a regular point if all components of $\gamma(t)$ are differentiable at $t$. As $\gamma(t)$ is Lipschitz continuous, almost every time $t \in \mathbb{R}_{+}$is a regular point. Since

$$
\beta(t)=\gamma(t)-\gamma(0)-\alpha t
$$


$\beta(t)$ is also differentiable at any regular point $t$ and

$$
\gamma^{\prime}(t)=\alpha-\beta^{\prime}(t)
$$

In the next, we derive the properties of $\beta^{\prime}(t)$.

Lemma 4.5: Consider a link $a \in A$. For any regular point $t>0$ with $\gamma_{a}(t)>0, \beta^{\prime}(t) \in \Phi_{a}$.

The proof of Lemma 4.5 is quite involved, and so are relegated to Appendix. Let $t_{0}=0$, and for each $1 \leq i \leq|A|$ let

$$
t_{i}=\sum_{j=1}^{i} \frac{1}{\left(1-\Delta^{\prec}(\alpha)\right)^{j}} .
$$

Lemma 4.6: For each $1 \leq i \leq|A|, \gamma_{a_{i}}(t)=0$ whenever $t \geq t_{i}$

Proof: We prove the lemma by contradiction. Assume to the contrary the lemma is false, and let $i$ be the smallest index between 1 and $|A|$ such that $\gamma_{a_{i}}(t)>0$ for some $t \geq t_{i}$. Consider any regular point $t>t_{i-1}$ with $\gamma_{a_{i}}(t)>0$. By Lemma $4.5, \beta^{\prime}(t) \in \Phi_{N_{G}^{\prec}\left(a_{i}\right)}$ and hence

$$
\Gamma\left(N_{G}^{\prec}\left(a_{i}\right), a_{i} ; \beta^{\prime}(t)\right) \geq 1 .
$$

Thus,

$$
\begin{aligned}
& \Gamma\left(N_{G}^{\prec}\left(a_{i}\right), a_{i} ; \gamma^{\prime}(t)\right) \\
& =\Gamma\left(N_{G}^{\prec}\left(a_{i}\right), a_{i} ; \alpha-\beta^{\prime}(t)\right) \\
& =\Gamma\left(N_{G}^{\prec}\left(a_{i}\right), a_{i} ; \alpha\right)-\Gamma\left(N_{G}^{\prec}\left(a_{i}\right), a_{i} ; \beta^{\prime}(t)\right) \\
& \leq \Delta^{\prec}(\alpha)-1 .
\end{aligned}
$$

On the other hand, by the choice of $i$,

$$
\begin{aligned}
& \Gamma\left(N_{G}^{\prec}\left(a_{i}\right), a_{i} ; \gamma^{\prime}(t)\right) \\
& =\sum_{b \in N_{G}^{\prec}\left(a_{i}\right)} c_{a_{i}, b} \gamma_{b}^{\prime}(t) \\
& =c_{a_{i}, a_{i}} \gamma_{a_{i}}^{\prime}(t) .
\end{aligned}
$$

Therefore,

$$
\gamma_{a_{i}}^{\prime}(t) \leq \frac{\Delta^{\prec}(\alpha)-1}{c_{a_{i}, a_{i}}}<0 .
$$

By Lemma 2.1 we have that $\gamma_{a_{i}}(t)=0$ for any

$$
\begin{aligned}
t & \geq t_{i-1}+\frac{\gamma_{a_{i}}\left(t_{i-1}\right)}{\frac{1-\Delta^{\prec}(\alpha)}{c_{a_{i}, a_{i}}}} \\
& =t_{i-1}+\frac{c_{a_{i}, a_{i}}}{1-\Delta_{i}^{\prec}(\alpha)} \gamma_{a_{i}}\left(t_{i-1}\right) .
\end{aligned}
$$

However, since

$$
\begin{aligned}
\gamma_{a_{i}}\left(t_{i-1}\right) & =\gamma_{a_{i}}(0)+\alpha_{a_{i}} t_{i-1}-\beta_{a_{i}}\left(t_{i-1}\right) \\
& \leq \gamma_{a_{i}}(0)+\alpha_{a_{i}} t_{i-1} \\
& \leq 1+\alpha_{a_{i}} t_{i-1},
\end{aligned}
$$

we have

$$
\begin{aligned}
& t_{i-1}+\frac{c_{a_{i}, a_{i}}}{1-\Delta^{\prec}(\alpha)} \gamma_{a_{i}}\left(t_{i-1}\right) \\
& \leq t_{i-1}+\frac{c_{a_{i}, a_{i}}}{1-\Delta^{\prec}(\alpha)}\left(1+\alpha_{a_{i}} t_{i-1}\right) \\
& =\frac{1-\Delta^{\prec}(\alpha)+c_{a_{i}, a_{i}} \alpha_{a_{i}}}{1-\Delta^{\prec}(\alpha)} t_{i-1}+\frac{c_{a_{i}, a_{i}}}{1-\Delta^{\prec}(\alpha)} \\
& \leq \frac{1}{1-\Delta^{\prec}(\alpha)} t_{i-1}+\frac{1}{1-\Delta^{\prec}(\alpha)} \\
& =t_{i},
\end{aligned}
$$

where the second inequality following from the fact that

$$
c_{a_{i}, a_{i}} \alpha_{a_{i}} \leq \Delta^{\prec}(\alpha)
$$

and

$$
c_{a_{i}, a_{i}} \leq 1
$$

Thus, $\gamma_{a_{i}}(t)=0$ for any $t \geq t_{i}$, which is a contradiction. So, the lemma holds.

Lemma 4.6 yields that $\gamma(t)=0$ for $t \geq t_{|A|}$. Since $T \geq$ $t_{|A|}$, we have

$$
\left\|\frac{z^{(n)}(n T)}{n}\right\|_{1} \rightarrow 0
$$

along the infinite sequence $S^{\prime}$. This completes the proof of Theorem 4.2.

\section{Throughrut EFFICIENCY RATIO}

In this section, we derive the lower bounds on the throughput efficiency ratio of a static GLS. Given a link ordering $\prec$ of $A$, its backward local independence number (BLIN) is defined to be

$$
\max _{a \in A} \max \left\{|I|: I \subseteq N_{G}^{\prec}(a), I \in \mathcal{I}\right\}
$$

Theorem 5.1: Consider a link ordering $\prec$ with BLIN $\mu$. Then $Q^{\prec} \supseteq \frac{1}{\mu+2} P$. In addition, for SC-SR wireless networks, $Q^{\prec} \supseteq \frac{1}{\mu} P$.

Proof: Consider any $d \in P$. By the first part of Lemma 2.7 in [21],

$$
\Delta^{\prec}(d) \leq(\mu+2) \chi^{*}(d)<\mu+2 .
$$

Thus, $d \in(\mu+2) Q^{\prec}$. Hence, $P \subseteq(\mu+2) Q^{\prec}$, which implies that $Q^{\prec} \supseteq \frac{1}{\mu+2} P$.

Next, we consider the special SC-SR setting. Consider any $d \in P$. By Corollary 5.2 in [14],

$$
\Delta^{\prec}(d) \leq \mu \chi^{*}(d)<\mu .
$$

Thus, $d \in \mu Q^{\prec}$. Hence, $P \subseteq \mu Q^{\prec}$, which implies that $Q^{\prec} \supseteq$ $\frac{1}{\mu} P$. 
Now, we apply the general Theorem 5.1 to two special static link orderings under the 802.11 interference model. The lexicographic ordering sorts all links in the lexicographic order of their left endpoints. The interference radius of a link is defined to be the larger one of the interference radii of its endpoints, and the interference radius decreasing ordering sorts all links in descending order of the interference radius. Since BLIN only depends on the topology of $G$ rather than the edge weight function $c$, the following properties which hold in the convectional conflict graph of the underlying SC-SR wireless network also hold in the $G$ :

- With uniform interference radii, the lexicographic ordering of $A$ has BLIN at most 6 [6].

- With arbitrary interference radii, the interference radius decreasing ordering of $A$ has BLIN at most 23 [14].

Theorem 4.1 and Theorem 5.1 immediately implies the following two corollaries.

Corollary 5.2: Under the 802.11 interference model with uniform interference radii, the throughput efficiency ratio of the static GLS in lexicographic ordering is at least $1 / 8$ in MC-MR wireless networks and at least $1 / 6$ in SC-SR wireless networks.

Corollary 5.3: Under the 802.11 interference model with arbitrary interference radii, the throughput efficiency ratio of the static GLS in interference radius decreasing ordering is at least $1 / 25$ in MC-MR wireless networks and at least $1 / 23$ in SC-SR wireless networks.

\section{DISCUSSIONS}

The static GLS in either the lexicographic ordering or the interference radius decreasing ordering has much implementation than the $\mathbf{L Q F}$ scheduling. In this section, we compare their stability performances.

For any $d \in \mathbb{R}_{+}^{A}$, let $\Delta^{*}(d)$ denote the smallest $d$-weighted inductivity of all possible link orderings of $A$. It was shown in [18] that

$$
Q^{*}=\left\{d \in \mathbb{R}_{+}^{A}: \Delta^{*}(d)<1\right\} .
$$

is a stability subregion of the $\mathbf{L Q F}$ scheduling. Consider any static link ordering $\prec$ of $A$. Since

$$
\Delta^{*}(d) \leq \Delta^{\prec}(d)
$$

for any $d \in \mathbb{R}_{+}^{A}$, we have $Q^{*} \supseteq Q^{\prec}$. Thus, the stability subregion $Q^{\prec}$ of the static GLS in the link ordering $\prec$ is also a stability subregion of the $\mathbf{L Q F}$ scheduling. On the other hand, the throughput efficiency ratio of the LQF scheduling under the 802.11 interference model has the following best-known lower bounds:

- With uniform interference radii, the throughput efficiency ratio of the $\mathbf{L Q F}$ scheduling is at least $1 / 8$ in MC-MR wireless networks [18] and exactly $1 / 6$ in SC-SR wireless networks [6].

- With arbitrary interference radii, the throughput efficiency ratio of the $\mathbf{L Q F}$ scheduling is at least $1 / 20$ in MC-MR wireless networks and at least $1 / 16$ in SC-SR wireless networks [18].

Thus, with uniform interference radii both the LQF scheduling and the static GLS in lexicographic ordering have the same best-known lower bounds on the throughput efficiency ratio by Corollary 5.2. With arbitrary interference radii, the LQF scheduling only has slightly better throughput efficiency ratio than the static GLS in interference radius decreasing ordering by Corollary 5.3. Therefore, with uniform (respectively, arbitrary) interference radii we advocate the static GLS in lexicographic ordering (respectively, interference radius decreasing ordering).

ACKNOWLEDGEMENTS: This work was supported in part by the National Science Foundation of USA under grants CNS-0831831, CNS-0916666, and CNS-1219109, by the National Natural Science Foundation of P. R. China under grants 61128005

\section{REFERENCES}

[1] A. Brzezinski, G. Zussman, and E. Modiano, Distributed throughput maximization in wireless mesh networks via pre-partitioning, IEEE/ACM Trans. Netw. 16(6): 1406-1419 (2008).

[2] P. Chaporkar, K. Kar, X. Luo, and S. Sarkar, Throughput and fairness guarantees through maximal scheduling in wireless networks, IEEE Transactions on Information Theory, vol. 54, no. 2, pp. 572-594, Feb. 2008.

[3] J. G. Dai, On Positive Harris Recurrence of Multiclass Queueing Networks: A Unified Approach via Fluid Limit Models, Annals of Applied Probability 5(1):49-77, 1995.

[4] A. Dimakis and J. Walrand, Sufficient conditions for stability of longest queue first scheduling: second order properties using fluid limits, Advances in Applied Probability, vol. 38, no. 2, pp. 505-521, June 2006.

[5] C. Joo, X. Lin, and N. B. Shroff, Performance limits of greedy maximal matching in multi-hop wireless networks, in Proc. IEEE CDC'07, Dec. 2007.

[6] C. Joo, X. Lin, and N. B. Shroff, Understanding the capacity region of the greedy maximal scheduling algorithm in multi-hop wireless networks, in Proc. IEEE INFOCOM 2008, April 2008.

[7] M. Leconte, J. Ni, and R. Srikant, Improved bounds on the throughput efficiency of greedy maximal scheduling in wireless networks, in Proc. ACM MOBIHOC'09, May 2009.

[8] B. Li, C. Boyaci, and Y. Xia, A refined performance characterization of longest-queue-first policy in wireless networks, in Proc. ACM MOBIHOC'09, May 2009. 
[9] X. Lin and S. Rasool, A Distributed Joint Channel-Assignment, Scheduling and Routing Algorithm for Multi-Channel Ad-hoc Wireless Networks, Proc. IEEE INFOCOM 2009, pp. 1118-1126.

[10] X. Lin and N. B. Shroff, The impact of imperfect scheduling on crosslayer rate control in wireless networks, IEEE/ACM Transactions on Networking, vol. 14, no. 2, pp. 302-315, Apr. 2006. The conference version appeared in Proceedings of IEEE INFOCOM, pages 1804-1814, 2005.

[11] V.A. Malyshev and M.V. Menshikov, Ergodicity, continuity and analyticity of countable Markov chains, Trans. Moscow Math. Soc. 39 1-48, 1981.

[12] A.V. Skorokhod, Basic principles and applications of probability theory, edited by Yu.V. Prokhorov, and translated from the 1989 Russian original by B.D. Seckler. Springer-Verlag, Berlin, 2005.

[13] L. Tassiulas and A. Ephremides, Stability properties of constrained queueing systems and scheduling policies for maximum throughput in multihop radio networks, IEEE Transactions on Automatic Control, vol. 37, no. 12, pp. 1936-1948, Dec. 1992.

[14] P.-J. Wan, Multiflows in Multihop Wireless Networks, ACM MOВIHOC 2009, pp. 85-94.

[15] P.-J. Wan, Y. Cheng, Z. Wang, and F. Yao, Multiflows in Multi-Channel Multi-Radio Multihop Wireless Networks, Proc. IEEE INFOCOM 2011, pp. 846-854.

[16] P.-J. Wan, M. Li, L. Wang, and O. Frieder, Local Pooling Factor of Multihop Wireless Networks, IEEE INFOCOM Mini-conference 2011.

[17] P.-J. Wan, C. Ma, Z. Wang, B. Xu, M. Li, and X. Jia, Weighted Wireless Link Scheduling without Information of Positions And Interference/Communication Radii, in Proc. IEEE INFOCOM 2011, pp. 2327-2335.

[18] P.-J. Wan, X.H. Xu, Z. Wang, S.-J. Tang, and Z.G. Wan, Stability Analyses of Longest-Queue-First Link Scheduling in MC-MR Wireless Networks, in Proc. ACM Mobihoc 2012.

[19] X. Wu and R. Srikant, Scheduling efficiency of distributed greedy scheduling algorithms in wireless networks, in Proceedings of IEEE INFOCOM, 2006.

[20] X. Wu, R. Srikant and J. R. Perkins, Queue-Length Stability of Maximal Greedy Schedules in Wireless Networks, IEEE Transactions on Mobile Computing, June 2007, pp. 595-605.

[21] P.-J. Wan, X. Jia, M. Li, H. Du, Z.G. Wan, and O. Frieder, Scalable Algorithms for Wireless Link Schedulings in Multi-Channel Multi-Radio Wireless Networks, submitted to IEEE INFOCOM 2013, available at http://www.cs.iit.edu/ ${ }^{\text {wan }} /$ ccg.pdf.

\section{APPENDIX}

In this appendix, we prove Lemma 4.5. Fix a regular point $t>0$ with $\gamma_{a}(t)>0$. Consider any $\varepsilon \in(0,1)$. Then, there exists $\delta>0$ such that for any $s \in(t, t+\delta]$,

$$
\gamma_{a}(s)>\frac{2}{3} \gamma_{a}(t)
$$

and

$$
\left\|\frac{\beta(s)-\beta(t)}{s-t}-\beta^{\prime}(t)\right\|_{\infty} \leq \varepsilon .
$$

Let $\varepsilon_{1}=\varepsilon /\|\tau\|_{\infty}$. By the u.o.c. convergence, there exists a sufficiently large $n \in S^{\prime}$ such that

$$
n \geq \max \left\{\frac{1}{\varepsilon_{1} \delta}, \frac{3}{\gamma_{a}(t)}\|\tau\|_{\infty}\right\}
$$

and for any $s \in[t, t+\delta]$,

$$
\begin{aligned}
\left\|\frac{y^{(n)}(n s)}{n}-\beta(s)\right\|_{\infty} & <\frac{\varepsilon}{2} \delta \\
\left|\frac{z_{a}^{(n)}(n s)}{n}-\gamma_{a}(s)\right| & <\frac{1}{3} \gamma_{a}(t) .
\end{aligned}
$$

We make the following two claims. $3 \varepsilon$.

Claim 6.1: $\left\|\bar{y}^{(n)}\left(n\left(t+\varepsilon_{1} \delta\right), n(t+\delta)\right)-\beta^{\prime}(t)\right\|_{\infty} \leq$

Proof: We first show that

$$
\left\|\bar{y}^{(n)}(n t, n(t+\delta))-\beta^{\prime}(t)\right\|_{\infty} \leq 2 \varepsilon,
$$

Since

$$
\begin{aligned}
& \left\|\bar{y}^{(n)}(n t, n(t+\delta))-(\beta(t+\delta)-\beta(t))\right\|_{\infty} \\
& =\left\|\frac{y^{(n)}(n(t+\delta))-y_{l}^{(n)}(n t)}{n}-(\beta(t+\delta)-\beta(t))\right\|_{\infty} \\
& \leq\left\|\frac{y^{(n)}(n(t+\delta))}{n}-\beta(t+\delta)\right\|_{\infty}+\left\|\frac{y^{(n)}(n t)}{n}-\beta(t)\right\|_{\infty} \\
& <\varepsilon \delta,
\end{aligned}
$$

we have

$$
\begin{aligned}
& \left\|\bar{y}^{(n)}(n t, n(t+\delta))-\frac{\beta(t+\delta)-\beta(t)}{\delta}\right\|_{\infty} \\
& =\left\|\frac{y^{(n)}(n(t+\delta))-y^{(n)}(n t)}{n \delta}-\frac{\beta(t+\delta)-\beta(t)}{\delta}\right\|_{\infty} \\
& <\varepsilon .
\end{aligned}
$$

Hence,

$$
\begin{aligned}
& \left\|\bar{y}^{(n)}(n t, n(t+\delta))-\beta^{\prime}(t)\right\|_{\infty} \\
\leq & \left\|\bar{y}^{(n)}(n t, n(t+\delta))-\frac{\beta(t+\delta)-\beta(t)}{\delta}\right\|_{\infty} \\
& +\left\|\frac{\beta(t+\delta)-\beta(t)}{\delta}-\beta^{\prime}(t)\right\|_{\infty} \\
< & 2 \varepsilon .
\end{aligned}
$$

Now, we show that

$$
\left\|\bar{y}^{(n)}(n t, n(t+\delta))-\bar{y}^{(n)}\left(n\left(t+\varepsilon_{1} \delta\right), n(t+\delta)\right)\right\|_{\infty} \leq \varepsilon .
$$

Since

$$
n\left(t+\varepsilon_{1} \delta\right)=n t+\varepsilon_{1}(n(t+\delta)-n t),
$$

by the first part of Lemma 4.4 we have

$$
\begin{aligned}
& \bar{y}^{(n)}(n t, n(t+\delta)) \\
= & \varepsilon_{1} \bar{y}^{(n)}\left(n t, n\left(t+\varepsilon_{1} \delta\right)\right)+ \\
& \left(1-\varepsilon_{1}\right) \bar{y}^{(n)}\left(n\left(t+\varepsilon_{1} \delta\right), n(t+\delta)\right) .
\end{aligned}
$$


Hence,

$$
\begin{aligned}
& \bar{y}^{(n)}(n t, n(t+\delta))-\bar{y}^{(n)}\left(n\left(t+\varepsilon_{1} \delta\right), n(t+\delta)\right) \\
& =\varepsilon_{1}\left(\bar{y}^{(n)}\left(n t, n\left(t+\varepsilon_{1} \delta\right)\right)-\bar{y}^{(n)}\left(n\left(t+\varepsilon_{1} \delta\right), n(t+\delta)\right)\right) .
\end{aligned}
$$

By the first part of Lemma 4.4,

$$
\begin{aligned}
& \left\|\bar{y}^{(n)}(n t, n(t+\delta))-\bar{y}^{(n)}\left(n\left(t+\varepsilon_{1} \delta\right), n(t+\delta)\right)\right\|_{\infty} \\
& \leq \varepsilon_{1} \max \left\{\begin{array}{c}
\left\|\bar{y}^{(n)}\left(n t, n\left(t+\varepsilon_{1} \delta\right)\right)\right\|_{\infty}, \\
\left\|\bar{y}^{(n)}\left(n\left(t+\varepsilon_{1} \delta\right), n(t+\delta)\right)\right\|_{\infty}
\end{array}\right\} \\
& \leq \varepsilon_{1}\|\tau\|_{\infty} \\
& =\varepsilon \text {. }
\end{aligned}
$$

The two inequalities shown above yield the inequality in the lemma immediately.

Claim 6.2: $\bar{y}^{(n)}\left(n\left(t+\varepsilon_{1} \delta\right), n(t+\delta)\right) \in \Phi_{a}$.

Proof: By the second part of Lemma 4.4, $\bar{y}^{(n)}\left(n\left(t+\varepsilon_{1} \delta\right), n(t+\delta)\right)$ is a convex combination of

$$
\left\{d^{(n)}(j):\left\lfloor n\left(t+\varepsilon_{1} \delta\right)\right\rfloor+1 \leq j \leq\lceil n(t+\delta)\rceil\right\} .
$$

Thus, it sufficient to show that $I^{(n)}(j)$ is $a$-tight for any integer $j$ between $\left\lfloor n\left(t+\varepsilon_{1} \delta\right)\right\rfloor+1$ and $\lceil n(t+\delta)\rceil$.

We first show that for any $s \in[t, t+\delta]$,

$$
z_{a}^{(n)}(n s)>\|\tau\|_{\infty} .
$$

Indeed, since

$$
\left|\frac{z_{a}^{(n)}(n s)}{n}-\gamma_{a}(s)\right|<\frac{1}{3} \gamma_{a}(t)
$$

Therefore,

$$
\frac{z_{a}^{(n)}(n s)}{n}>\gamma_{a}(s)-\frac{1}{3} \gamma_{a}(t)>\frac{1}{3} \gamma_{a}(t),
$$

which implies

$$
z_{a}^{(n)}(n s)>\frac{1}{3} \gamma_{a}(t) n \geq\|\tau\|_{\infty} .
$$

So, the desired inequality holds.
Consider any integer $j$ between $\left\lfloor n\left(t+\varepsilon_{1} \delta\right)\right\rfloor+1$ and $\lceil n(t+\delta)\rceil$. Since

$$
\begin{aligned}
& \left\lfloor n\left(t+\varepsilon_{1} \delta\right)\right\rfloor-n t \\
& >n\left(t+\varepsilon_{1} \delta\right)-1-n t \\
& =n \varepsilon_{1} \delta-1 \\
& \geq 0
\end{aligned}
$$

and

$$
\lceil n(t+\delta)\rceil-1<n(t+\delta)
$$

we have

$$
n t \leq\left\lfloor n\left(t+\varepsilon_{1} \delta\right)\right\rfloor \leq j-1 \leq\lceil n(t+\delta)\rceil-1<n(t+\delta)
$$

Thus, at the end of the $(j-1)$-th times-slot, the queue length of the link $a$ is is greater than $\|\tau\|_{\infty}$. Thus, $I^{(n)}(j)$ is $a$-tight.

Since $\Phi_{a}$ is compact, the above two claims together with fact that $\varepsilon$ can be chosen arbitrarily small implies the correctness of Lemma 4.5. 